\title{
Germinación de semillas de Albizia hassleri a diferentes temperaturas, en condiciones de laboratorio
}

\author{
Germination of Albizia hassleri seeds at different temperatures, under laboratory conditions \\ José Luis Soto Gonzales ${ }^{\mathrm{a}^{*}}$, Sérgio Valiengo Valeri ${ }^{\mathrm{b}}$, Rinaldo César de Paula \\ *Autor de correspondencia: aUniversidad Estadual Paulista, UNESP/FCAV, Jaboticabal, San Pablo, Brasil, \\ tel.11-96184922, jolusogol@hotmail.com \\ bUniversidad Estadual Paulista, UNESP-FCAV, Jaboticabal, San Pablo, Brasil.
}

\begin{abstract}
SUMMARY
The objective of this study was to analyze the germination of seeds of Albizia hassleri under different temperatures. A completely random design arranged as a split plot for temperatures regimes, with 11 seed lots and four replications of 15 seeds was used. The plot was represented by the various lots and the sub plots for different temperatures. The means were compared by Scott-Knott test at 5\% probability. The temperatures used were: a) constant: 20,25 and $30^{\circ} \mathrm{C}$, and b) alternating: $20-30$ and $25-35^{\circ} \mathrm{C}$. For all 11 seed lots the mean germination was $90 \%$, speed germination index (IVG) was 5.059, fresh matter of seedlings (MMF) was 0.0628 $\mathrm{g}$ and dry matter (MMS) $0.0499 \mathrm{~g}$. The variation coefficient (CV) between plots ranged from $8.48 \%$ for germination to $51.71 \%$ for dry matter of seedlings and sub plot of $6.77 \%$ to $60.45 \%$ for germination and MMS. These high values of CV, tested for MMS and MMF, indicate low repeatability of results within each treatment. In general, the IVG obtained at temperatures of 20 and $25^{\circ} \mathrm{C}$ was lower than those in temperatures of $30,20-30$ and $25-35^{\circ} \mathrm{C}$. The best temperature for IVG was the alternating $25-35^{\circ} \mathrm{C}$ and constant $30^{\circ} \mathrm{C}$. The germination test can be conducted at $30,20-30$ and $25-35^{\circ} \mathrm{C}$ for 19 days.
\end{abstract}

Key words: forest seeds, native species, germination temperatures.

\section{RESUMEN}

El objetivo de este estudio fue analizar el comportamiento germinativo de semillas de Albizia hasslerii bajo diferentes temperaturas. El diseño estadístico utilizado fue completamente al azar en parcelas subdivididas para temperaturas, con 11 árboles madres llamados lotes y cuatro repeticiones de 15 semillas. La parcela fue representada por los diferentes lotes y las subparcelas por las diferentes temperaturas. La comparación de las medias fue realizada por la prueba de Scott-Knott al 5\% de probabilidad. Las temperaturas utilizadas fueron: a) constantes: 20,25 y $30^{\circ} \mathrm{C}$; y b) alternadas: 20-30 y 25-35 $\mathrm{C}$. Para el conjunto de los 11 lotes se obtuvo germinación media de 90\%, índice de velocidad de germinación (IVG) de 5,059, masa de materia fresca (MMF) de

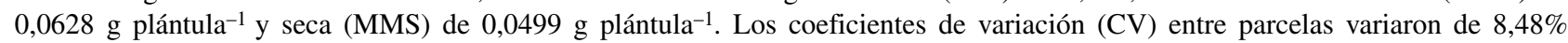
para germinación a $51,71 \%$ para masa seca de plántula y subparcela de 6,77\% para germinación a 60,45\% para MMS. Estos altos valores de CV, verificados para MMF y MMS indican baja repetibilidad de los resultados dentro de cada tratamiento. En general, los índices de velocidad de germinación (IVG) obtenidos en las temperaturas de 20 y $25^{\circ} \mathrm{C}$ fueron inferiores a los obtenidos en las temperaturas de $30,20-30$ y $25-35^{\circ} \mathrm{C}$. La mejor temperatura para IVG fue la alternada de $25-35^{\circ} \mathrm{C}$ y la constante de $30^{\circ} \mathrm{C}$. El ensayo de germinación puede ser realizado a 30, 20-30 y $25-35^{\circ} \mathrm{C}$, por 19 días.

Palabras clave: semilla forestal, especie nativa, temperaturas de germinación.

\section{INTRODUCCIÓN}

Albizia hassleri (Chodat) Burr. pertenece a la familia Fabaceae-Mimosoideae. Frecuente en los bosques brasileros, principalmente en la región de la Floresta Atlántica. Esta especie forestal produce anualmente una gran cantidad de semillas, que deben ser puestas a germinar después de haber sido recolectadas, debido a su corta viabilidad. Sus principales características son el tronco liso y claro, floración blanca a crema, con flores muy pequeñas (Lorenzi 1992). Su madera es leve, suave al corte, poco compacta, de baja resistencia al ataque de organismos xilófagos. Su altura puede llegar a $20 \mathrm{~m}$, con tronco de hasta $60 \mathrm{~cm}$ de diámetro. Es empleada en obras internas de construcción civil, como también en la fabricación de forros, tablas, cajas y en la confección de objetos leves como lápices (Lorenzi 1992).

Esta especie arbórea nativa del Brasil se encuentra en la lista de especies amenazadas; está indicada para recomponer áreas degradadas y para uso en paisajismo por poseer cualidades ornamentales. Sin embargo, como para la mayoría de las especies nativas, son escasos los estudios. 
Uno de los medios utilizados para determinar el nivel de calidad de las semillas es el ensayo de germinación, el cual es realizado bajo condiciones de temperatura y sustratos ideales para cada especie (Gomes y Bruno 1992). Estudios sobre la influencia de la temperatura en la germinación de semillas son esenciales para entender los procesos bioquímicos y ecofisiológicos (Labouriau 1983, Ferreira y Borghetti 2004), los efectos pueden ser evaluados a partir de los cambios causados en el porcentaje, la velocidad y la frecuencia relativa de la germinación en el tiempo de incubación (Labouriau y Pacheco 1978). De acuerdo con Labouriau (1983), el rango de temperaturas apropiadas es aquel donde ocurre el máximo de germinabilidad, registrando el más alto porcentaje de germinación en corto tiempo.

Las semillas de diferentes especies tienen comportamientos variables respecto a la temperatura de germinación, lo cual puede proporcionar información de interés biológico y ecológico (Labouriau 1983). Dentro de una gama de temperaturas en las que las semillas de una especie germinan, hay una temperatura óptima, conocida como en la que ocurre el máximo de germinación y ésta se produce en un intervalo menor de tiempo. Las temperaturas mínimas y máximas son aquellas en las que la germinación es cero (Mayer y Poljakoff-Mayber 1989, Borges y Rena 1993).

Según Copeland y McDonald (1995), determinadas especies presentan mejor comportamiento germinativo cuando están expuestas a la alternancia de temperaturas. Ésta corresponde a las fluctuaciones que se encuentran en el entorno natural del bosque. Existen especies en que la germinación de sus semillas se ve favorecida cuando éstas son sometidas a temperaturas constantes (Lima et al. 1997), otras exigen alternancia de temperatura (Salomão et al. 1995). También, especies que son indiferentes a temperaturas constantes o alternadas en la germinación (Albuquerque et al. 1998). Pereira y Andrade (1994) recomiendan el uso de temperaturas alternadas con rangos de $20-30^{\circ} \mathrm{C}$ o $15-35^{\circ} \mathrm{C}$, sobre vermiculita, papel filtro o papel toalla para el ensayo de germinación de semillas de Psidium guajava $\mathrm{L}$.

El presente trabajo tuvo como objetivo determinar la temperatura y el tiempo (duración) para la conducción del ensayo de germinación de las semillas en condiciones de laboratorio.

\section{MÉTODOS}

Fueron seleccionados 11 árboles madre, cada uno representando un lote de semillas, según criterios establecidos por Capelanes y Biella (1984), teniendo en consideración los siguientes aspectos: buen aspecto fitosanitario (dentro de los cuales se destacaron árboles vigorosos, libres de plagas y enfermedades); con buena producción de semillas y árboles no aislados.
El experimento de germinación fue realizado en cámaras verticales, tipo B.O.D., con fotoperíodo de $8 \mathrm{~h}$. Se ensayaron las siguientes temperaturas de germinación: a) constantes: 20,25 y $30^{\circ} \mathrm{C}$; y b) alternadas: $20-30$ y $25-35^{\circ}$ C. En el caso de la temperatura alternada el período luminoso correspondió a las temperaturas alternadas de 30 y $35^{\circ} \mathrm{C}$.

Se usaron 15 semillas por repetición por cada árbol, las cuales fueron previamente desinfectadas por inmersión en solución de hipoclorito de sodio (2\%), por tres minutos, con posterior lavado en agua destilada. Las semillas fueron distribuidas en cajas transparentes de plástico, con tapas ("gerbox"), de $11 \times 11 \times 4 \mathrm{~cm}$, sobre dos hojas de papel filtro previamente esterilizadas en estufa a $105^{\circ} \mathrm{C}$ por 2 horas, conforme a recomendaciones de las Reglas de Análisis de Semillas (Ministério da Agricultura e Reforma Agrária 1992), y humedecidas con agua destilada (volumen de agua equivalente a 2,5 veces el peso del papel). Los "gerbox" fueron sellados con plástico transparente con el propósito de evitar la pérdida de agua por evaporación y rehumedecimiento del sustrato durante el experimento.

Las evaluaciones del número de semillas germinadas fueron efectuadas diariamente, hasta la estabilización de la germinación que duró 19 días. Los resultados fueron expresados en porcentaje de plántulas normales (Ministério da Agricultura e Reforma Agrária 1992), índice de velocidad de germinación (Maguire 1962) y masa de materia fresca y seca de plántulas.

La interpretación del ensayo de germinación fue efectuada en base a los criterios generales establecidos en las Reglas de Análisis de Semillas (Ministério da Agricultura e Reforma Agrária 1992), siendo consideradas como plantas normales aquellas con todas las estructuras esenciales o perfectas (Oliveira 1993).

La masa de materia fresca de las plántulas normales fue determinada en balanza analítica con precisión de $0,001 \mathrm{~g}$ y la materia seca fue evaluada después de someter las plántulas normales a secado en estufa de circulación de aire a $70^{\circ} \mathrm{C}$ por 48 horas.

El experimento fue instalado en un diseño íntegramente aleatorizado, en parcelas subdivididas para temperaturas, con cuatro repeticiones de 15 semillas. La parcela fue representada por los diferentes árboles y las subparcelas por las diferentes temperaturas. Los datos de porcentaje de plántulas normales fueron sometidos a la prueba de normalidad de Lilliefors (Cruz 2001), pero no hubo la necesidad de transformación de estos datos. Las medias entre tratamientos fueron discriminadas por la prueba de agrupamiento de Scott y Knott (1974).

\section{RESULTADOS}

Hubo efectos significativos $(P \leq 0,01)$ de árboles, temperaturas y de interacción árboles $\mathrm{x}$ temperaturas para la 
germinación e índice de velocidad de germinación (IVG); para masa de materia fresca (MMF) y masa de materia seca (MMS) de plántulas hubo efectos significativos de árboles y de temperaturas $(P \leq 0,05)$ y no significativo $(P>0,05)$ de interacción entre estos factores (cuadro 1$)$.

En el conjunto de los 11 árboles estudiados la germinación media fue de 90,2\%, IVG de 5,059, MMF de 0,0628 g plántula ${ }^{-1}$ y MS de 0,0499 g plántula $^{-1}$. Los coeficientes de variación (CV) entre parcelas variaron de $8,5 \%$, para germinación, a $51,7 \%$ para masa de materia seca de plántula y la subparcela de $6,8 \%$ para germinación a 60,5\% para MMS. Estos altos valores de CV, verificados para MMF y MMS indican baja repetitibilidad de los resultados dentro de cada tratamiento, dificultando la detección de diferencias entre los mismos.

Hubo buen porcentaje de germinación en todas las temperaturas ensayadas, en tanto la temperatura de $20^{\circ} \mathrm{C}$ proporcionó de modo general menores valores de germinación (cuadro 2). En esta temperatura los árboles quedaron divididos en tres grupos. En el primero están reunidos los árboles 3, 5, 7, 8, 9 y 11 con germinación variando de 90\%, para los árboles 9 y 5, a $98 \%$ para el árbol 8; en el segundo grupo se encuentran los árboles 2, 4, 6 y 10 , con valores de germinación de $82 \%$ para los árboles 2 y 6, a $87 \%$ para el árbol 4; el tercer grupo fue formado por el árbol 1 con $53 \%$ de germinación.

Cuadro 1. Resumen de análisis de varianza para las características de germinación (G), índice de velocidad de germinación (IVG), masa de materia fresca (MMF, g plántula ${ }^{-1}$ ) y masa de materia seca (MMS, g plántula ${ }^{-1}$ ) obtenidas de semillas de Albizia hassleri, provenientes de diferentes árboles, y sometidas a diferentes temperaturas de germinación.

Summary of analysis of variance for germination (\%G), germination speed index (IVG), fresh matter (MMF, $\mathrm{g}^{-1}$ seedling) and dry matter (MMS, g seedling ${ }^{-1}$ ) obtained from Albizia hassleri-seeds, from different mother trees (seed lots), and subjected to different germination temperatures.

\begin{tabular}{|c|c|c|c|c|c|}
\hline \multirow{2}{*}{$\begin{array}{l}\text { Fuentes de } \\
\text { variación }\end{array}$} & \multirow{2}{*}{$\mathrm{gl}$} & \multicolumn{4}{|c|}{ Cuadrados medios } \\
\hline & & $\% \mathrm{G}$ & IVG & MMF & MMS \\
\hline Árboles (M) & 10 & $1.136,0 * *$ & $39,511 * *$ & $0,0024 * *$ & $0,0014^{*}$ \\
\hline Error (A) & 33 & 58,6 & 0,268 & 0,0006 & 0,0006 \\
\hline Temperatura $(\mathrm{T})$ & 4 & $208,4 * *$ & $16,895^{* *}$ & $0,0029^{*}$ & $0,0026^{*}$ \\
\hline $\mathrm{M} \times \mathrm{T}$ & 40 & $110,9 * *$ & $1,437 * *$ & $0,0011 \mathrm{~ns}$ & $0,0006 \mathrm{~ns}$ \\
\hline Error (B) & 132 & 37,4 & 0,40 & 0,0009 & 0,0009 \\
\hline Media & 219 & 90,2 & 5,059 & 0,0628 & 0,0499 \\
\hline CV Parcela & 10,2 & 8,5 & 10,2 & 39,3 & 51,7 \\
\hline CV Subparcela & 12,5 & 6,8 & 12,5 & 48,4 & 60,5 \\
\hline
\end{tabular}

gl: grados de libertad . CV: coeficiente de variación (\%). ns: no significativo $(P>0,05)$. ${ }^{*} P<0,05$. $* * P<0,01$.

Cuadro 2. Desdoblamiento de la interacción árboles x temperaturas (A x T) para porcentaje de germinación e índice de velocidad de germinación (IVG), obtenidos de semillas de Albizia hassleri provenientes de 11 árboles y sometidas a diferentes temperaturas de germinación.

Splitting of the interaction mother trees (seed lots) arrays $\mathrm{x}$ temperatures (A x T) for germination percentage and speed germination index (IVG), derived from Albizia hassleri-seeds, from 11 seed lots and subjected to different germination temperatures.

\begin{tabular}{|c|c|c|c|c|c|c|c|c|c|c|}
\hline \multirow{2}{*}{ Árboles } & \multicolumn{5}{|c|}{ Porcentaje de germinación ${ }^{1}$} & \multicolumn{5}{|c|}{ Índice de velocidad de germinación (IVG) ${ }^{1}$} \\
\hline & $20^{\circ} \mathrm{C}$ & $25^{\circ} \mathrm{C}$ & $30^{\circ} \mathrm{C}$ & $20-30^{\circ} \mathrm{C}$ & $25-35^{\circ} \mathrm{C}$ & $20^{\circ} \mathrm{C}$ & $25^{\circ} \mathrm{C}$ & $30^{\circ} \mathrm{C}$ & $20-30^{\circ} \mathrm{C}$ & $25-35^{\circ} \mathrm{C}$ \\
\hline 2 & $53 \mathrm{bC}$ & $72 \mathrm{aD}$ & $80 \mathrm{aB}$ & $75 \mathrm{aB}$ & $73 \mathrm{aB}$ & $0,77 \mathrm{bF}$ & $1,53 \mathrm{bD}$ & $2,71 \mathrm{aE}$ & $2,02 \mathrm{aC}$ & $2,30 \mathrm{aD}$ \\
\hline 3 & $82 \mathrm{aB}$ & $88 \mathrm{aB}$ & $90 \mathrm{aA}$ & $87 \mathrm{aA}$ & $95 \mathrm{aA}$ & $2,97 \mathrm{bE}$ & $3,71 \mathrm{bC}$ & $5,06 \mathrm{aC}$ & $4,25 \mathrm{aB}$ & $4,66 \mathrm{aC}$ \\
\hline 4 & $97 \mathrm{aA}$ & $100 \mathrm{aA}$ & $98 \mathrm{aA}$ & $95 \mathrm{aA}$ & $100 \mathrm{aA}$ & $5,75 b B$ & $7,30 \mathrm{aA}$ & $6,62 \mathrm{aA}$ & $5,96 \mathrm{bA}$ & $7,40 \mathrm{aA}$ \\
\hline 5 & $87 \mathrm{bB}$ & $100 \mathrm{aA}$ & $93 \mathrm{aA}$ & $95 \mathrm{aA}$ & $100 \mathrm{aA}$ & $2,78 \mathrm{cE}$ & $4,62 \mathrm{bC}$ & $4,20 \mathrm{bD}$ & $4,63 \mathrm{cB}$ & $6,51 \mathrm{aA}$ \\
\hline 6 & $90 \mathrm{aA}$ & $92 \mathrm{aB}$ & $97 \mathrm{aA}$ & $92 \mathrm{aA}$ & $87 \mathrm{aA}$ & $6,15 \mathrm{aB}$ & $6,48 \mathrm{aA}$ & 7,18aA & $6,64 \mathrm{aA}$ & $6,35 \mathrm{aA}$ \\
\hline 7 & $82 \mathrm{bB}$ & $83 b C$ & $93 \mathrm{aA}$ & $88 \mathrm{aA}$ & $92 \mathrm{aA}$ & $2,43 \mathrm{bE}$ & $4,63 \mathrm{aC}$ & $5,34 \mathrm{aC}$ & $4,24 \mathrm{aB}$ & $5,00 \mathrm{aC}$ \\
\hline 9 & $97 \mathrm{aA}$ & $100 \mathrm{aA}$ & $92 \mathrm{aA}$ & $93 \mathrm{aA}$ & $93 \mathrm{aA}$ & $4,70 \mathrm{bC}$ & $5,07 \mathrm{bC}$ & $5,66 \mathrm{aB}$ & $4,90 \mathrm{bB}$ & $6,16 \mathrm{aA}$ \\
\hline 10 & $98 \mathrm{aA}$ & $100 \mathrm{aA}$ & $97 \mathrm{aA}$ & $100 \mathrm{aA}$ & $97 \mathrm{aA}$ & $4,92 \mathrm{bC}$ & $4,60 \mathrm{bC}$ & $6,63 \mathrm{aA}$ & $4,94 \mathrm{bB}$ & $6,92 \mathrm{aA}$ \\
\hline 11 & $90 \mathrm{aA}$ & $90 \mathrm{aB}$ & $85 \mathrm{aB}$ & $88 \mathrm{aA}$ & $92 \mathrm{aA}$ & $4,88 \mathrm{bC}$ & $5,51 \mathrm{bB}$ & $6,16 \mathrm{aB}$ & $4,85 \mathrm{bB}$ & $6,50 \mathrm{aA}$ \\
\hline 13 & $85 \mathrm{bB}$ & $93 \mathrm{aB}$ & $80 \mathrm{bB}$ & $97 \mathrm{aA}$ & $92 \mathrm{aA}$ & $3,64 \mathrm{bD}$ & $4,32 \mathrm{bC}$ & $4,94 \mathrm{aC}$ & $4,24 \mathrm{bB}$ & $5,45 \mathrm{aB}$ \\
\hline 14 & $93 \mathrm{bA}$ & $100 \mathrm{aA}$ & $88 \mathrm{bA}$ & $90 \mathrm{bA}$ & $78 \mathrm{cB}$ & $6,91 \mathrm{aA}$ & 7,09aA & $6,62 \mathrm{aA}$ & $6,56 \mathrm{aA}$ & $5,79 \mathrm{bB}$ \\
\hline
\end{tabular}

1 Medias seguidas por la misma letra, minúscula en la línea y mayúscula en la columna, no difieren entre sí por la prueba de Scott y Knott $(P>0,05)$. 
Los árboles fueron agrupados en cuatro grupos en la temperatura de $25^{\circ} \mathrm{C}$. Los árboles 4, 5, 9, 10 y 14 formaron el primer grupo con $100 \%$ de germinación; el segundo grupo estuvo compuesto por los árboles 3, 6, 11 y 13 con germinación, variando de $88 \%$ para el árbol 3, a 93\% para el árbol 13; el árbol 7 formó el tercer grupo con $83 \%$ de germinación y el árbol 2, con $72 \%$ de germinación, formó el último grupo.

La germinación a $30^{\circ} \mathrm{C}$ diferenció los árboles en dos grupos, el primero estuvo formado por los árboles 3, 4, $5,6,7,9,10$ y 14 con valores de germinación variando de 88\%, para el árbol 14, a 98\% para el árbol 4; en el segundo grupo aparecen los árboles 2,11 y 13 con germinación variando de $80 \%$, para los árboles 2 y 13 , a $85 \%$ para el árbol 11. En las temperaturas de $20-30^{\circ} \mathrm{C}$, el árbol 2 se observó nuevamente con peor desempeño, con $75 \%$ de germinación, siendo superado por los demás árboles, los cuales forman el primer grupo, con germinación variando de 87\% para el árbol 3, a 100\% para el árbol 10. A temperaturas de $25-35^{\circ} \mathrm{C}$ el árbol 2, con germinación de $73 \%$, y el árbol 14, con 78\%, no difieren entre sí y fueron superados por el resto de los árboles madres, cuya germinación varió de 87\%, para el árbol 6, a 100\% para los árboles 4 y 5.

En cuanto al efecto de las temperaturas, se observó que hay un comportamiento diferenciado para cada árbol en general. La temperatura de $20^{\circ} \mathrm{C}$ proporcionó menor porcentaje de germinación y las temperaturas de 30, 20-30 y $25-35^{\circ} \mathrm{C}$ posibilitaron mayor desempeño germinativo (cuadro 2).

La mejor temperatura para el IVG fue la alternada de $25-35^{\circ} \mathrm{C}$ y la constante de $30^{\circ} \mathrm{C}$. El número de grupos formados por árboles en las diferentes temperaturas fue de seis grupos a $20^{\circ} \mathrm{C}$, cuatro grupos a 25 y $25-35^{\circ} \mathrm{C}$, cinco grupos a $30^{\circ} \mathrm{C}$ y tres grupos a $20-30^{\circ} \mathrm{C}$. Estos resultados demuestran un comportamiento diferenciado de los árboles frente a la temperatura e indican que hay una temperatura o un rango de temperaturas óptimos para el proceso germinativo.

La masa de materia fresca (MMF) y la masa de materia seca (MMS) de plántulas presentaron el mismo comportamiento entre los árboles, donde los árboles 4, 6, 9, 10 y 14 fueron superados por los demás árboles. Por otro lado, evaluando el efecto de las temperaturas sobre estas características, las temperaturas de 25,30 y $20-30^{\circ} \mathrm{C}$ proporcionaron mayor MMF que las temperaturas de $20 \mathrm{y}$ 25-35 $\mathrm{C}$; para MMS la temperatura alternada de $25-35^{\circ} \mathrm{C}$ proporcionó un desempeño inferior en relación a las demás temperaturas estudiadas (cuadro 3 ).

\section{DISCUSIÓN}

Según Hendricks y Taylorson (1976) las bajas temperaturas pueden originar disminución de las tasas metabólicas hasta el punto en que los procesos esenciales para la
Cuadro 3. Medias para masa de materia fresca (MMF) y masa de materia seca (MMS) de plántulas obtenidas a partir de semillas de 11 árboles de Albizia hassleri y sometidas a diferentes temperaturas de germinación.

Fresh (MMF) and dry (MMS) matter weight of seedlings obtained from seeds of 11 mother trees (seed lots) of Albizia hassleriseeds and submitted to different germination temperatures.

\begin{tabular}{ccc}
\hline Árboles & MMF & MMS \\
\hline 2 & $0,075 \mathrm{a}$ & $0,058 \mathrm{a}$ \\
3 & $0,070 \mathrm{a}$ & $0,054 \mathrm{a}$ \\
4 & $0,047 \mathrm{~b}$ & $0,038 \mathrm{~b}$ \\
5 & $0,077 \mathrm{a}$ & $0,059 \mathrm{a}$ \\
6 & $0,055 \mathrm{~b}$ & $0,042 \mathrm{~b}$ \\
7 & $0,065 \mathrm{a}$ & $0,052 \mathrm{a}$ \\
9 & $0,048 \mathrm{~b}$ & $0,037 \mathrm{~b}$ \\
10 & $0,059 \mathrm{~b}$ & $0,046 \mathrm{~b}$ \\
11 & $0,068 \mathrm{a}$ & $0,052 \mathrm{a}$ \\
13 & $0,074 \mathrm{a}$ & $0,059 \mathrm{a}$ \\
14 & $0,054 \mathrm{~b}$ & $0,042 \mathrm{~b}$ \\
\hline Temperaturas $\left({ }^{\circ} \mathrm{C}\right)$ & & \\
\hline 20 & $0,058 \mathrm{~B}$ & $0,050 \mathrm{~A}$ \\
25 & $0,071 \mathrm{~A}$ & $0,050 \mathrm{~A}$ \\
30 & $0,069 \mathrm{~A}$ & $0,057 \mathrm{~A}$ \\
$20-30$ & $0,064 \mathrm{~A}$ & $0,051 \mathrm{~A}$ \\
$25-35$ & $0,051 \mathrm{~B}$ & $0,036 \mathrm{~B}$ \\
\hline
\end{tabular}

Medias seguidas por una misma letra minúscula para árboles y mayúscula para temperaturas, no difieren entre sí por la prueba de Scott y Knott $(P>0,05)$.

germinación dejan de ocurrir, como fue verificado para la temperatura de $20^{\circ} \mathrm{C}$, donde fueron observados los más bajos porcentajes para germinación. Con temperaturas más bajas, dada la reducción del metabolismo, la semilla puede germinar en períodos más largos (Amaral y Paulilo 1992). Por otro lado, dentro de ciertos límites, a temperaturas elevadas, la velocidad de absorción de agua y de las reacciones químicas es mayor, y las semillas germinan más rápidamente (Carvalho y Nakagawa 2000). Estos autores señalan que la temperatura óptima para germinación total es diferente de la óptima para velocidad de germinación de las semillas.

Según Bewley y Black (1985), la alternancia de temperaturas beneficia la ruptura de la dormancia y, consecuentemente, el proceso germinativo, siendo este acontecimiento más común para especies no domesticadas y de estadios iniciales de sucesión. Santos y Aguiar (2000) observaron que la temperatura alternada promovió mayor porcentaje y velocidad de germinación en semillas de Sebastiania commersoniana (Baill.) L. B. Smith et R. J. Downs. Como se pudo verificar en este trabajo, cuando fueron usadas temperaturas alternadas se obtuvieron mayores porcentajes de germinación.

Resultados semejantes a lo observado para germinación ocurrieron para IVG (cuadro 2). En general, el índice de velocidad de germinación (IVG) obtenido en las temperaturas 
de 20 y $25^{\circ} \mathrm{C}$ fue inferior a los obtenidos en las temperaturas de $30^{\circ} \mathrm{C}, 20-30^{\circ} \mathrm{C}$ y $25-35^{\circ} \mathrm{C}$ (cuadro 2). Según Carvalho y Nakagawa (2000) temperaturas bajo la óptima tienden a reducir la velocidad del proceso germinativo, exponiendo las plántulas por mayor período de tiempo a factores adversos, pudiendo llevar a la reducción total de la germinación.

Kraemer et al. (2000), estudiando la germinación de semillas de Tibouchina urvilleana (DC.) Cogn., constataron mayor velocidad y homogeneidad en la germinación a $25^{\circ} \mathrm{C}$, disminuyendo en las temperaturas sobre y bajo ésta. Bajo $10^{\circ} \mathrm{C}$ no ocurrió germinación. Alves et al. (2002) relatan que la temperatura de $25^{\circ} \mathrm{C}$ se mostró más adecuada para la conducción del ensayo de germinación en semillas de Mimosa caesalpiniaefolia Benth., independiente del sustrato utilizado. Araújo Neto et al. (2003) constataron que la temperatura constante de $25^{\circ} \mathrm{C}$ fue la más apropiada para que ocurra germinación en semillas de Acacia polyphylla DC.

Semillas de algunas especies tropicales respondieron mejor al régimen de temperatura alternada en comparación con el régimen de temperatura constante (Vazquez-Yanes y Orozco-Segovia 1982, Probert et al. 1986). Para Mayer y Poljakoff-Mayber (1989) la alternancia de temperatura hace que la germinación de las semillas aumente en relación a cada temperatura; según Hand et al. (1982) esta fluctuación actúa directamente sobre la permeabilidad de las membranas, permitiendo así que la germinación ocurra. De acuerdo con Vazquez-Yanes y Orozco-Segovia (1984), esa alternancia puede estar actuando sobre mecanismos enzimáticos, desencadenando la germinación en las semillas.

De acuerdo con Borges y Rena (1993), la temperatura de 20 a $30^{\circ} \mathrm{C}$ se muestra adecuada para la germinación de semillas de la mayoría de las especies tropicales. Paula (2007) comenta que, en general, la evaluación de la masa de materia fresca y seca de plántulas no presenta resultados satisfactorios para la discriminación de tratamientos en ensayos de germinación de semillas forestales, como fue verificado en este trabajo, donde la masa de materia fresca y seca no demostró resultados satisfactorios en la discriminación de los tratamientos. De acuerdo con Nakagawa (1999), lotes con menor germinación tienden a presentar mayores valores de masa de materia seca de plántulas que lotes con mayor germinación, tal vez por ocupar mayor espacio en las cajas de germinación.

En un estudio realizado por Cavalcante y Perez (1995) las especies tropicales presentan una notable tolerancia a altas temperaturas, mostrando generalmente un límite máximo $\geq 35^{\circ} \mathrm{C}$, en tanto éstas son sensibles a temperaturas bajas, presentando generalmente límite inferior sobre $5^{\circ} \mathrm{C}$. Castro (2003), estudiando semillas de Tabebuia capitata (Bureau et Schum.), verifica que con las temperaturas de 30 y $35^{\circ} \mathrm{C}$ obtuvo los mayores porcentajes de germinación, tanto para la emisión de la raíz primaria como para formación de plántulas normales.

\section{CONCLUSIONES}

Para semillas de Albizia hassleri provenientes de diferentes árboles madre se puede concluir que: a) se encontró gran variabilidad entre los lotes de semillas de los árboles madre en cuanto al proceso germinativo y b) la alternancia de temperaturas favorece el proceso de germinación de estas semillas.

\section{AGRADECIMIENTOS}

El primer autor desea expresar sus sinceros agradecimientos a la Coordinación de Perfeccionamiento de Personas de Nivel Superior (CAPES), por la beca concedida para la realización de la presente investigación.

\section{REFERENCIAS}

Albuquerque MCF, TJD Rodrigues, L Minohara, ND Tebaldi, LMM Silva. 1998. Influência da temperatura e do substrato na germinação de sementes de saguaragi (Colubrina glandulosa Perk)- Rhamanaceae. Revista Brasileira de Sementes 20(2): 346-349.

Alves EU, RC Paula, AP Oliveira, RLA Bruno, AA Diniz. 2002. Germinação de sementes de Mimosa caesalpiniaefolia Benth. em diferentes substratos e temperaturas. Revista Brasileira de Sementes 24(1): 169-178.

Amaral LIV, MTF Paulilo. 1992. Efeito da luz, temperatura, regulador de crescimento e nitrato de potássio na germinação de Miconia cinnamomifolia Naudim. Insula 21: 59-86.

Araújo Neto JC, IB Aguiar, VM Ferreira. 2003. Efeito da temperatura e da luz na germinação de sementes de Acacia polyphylla D.C. Revista Brasileira de Botânica 26(2): 249-256.

Bewley JD, M Black. 1985. Seeds: physiology of development and germination. New York, USA. Plenum Press. p. 237-252.

Borges EEI, AB Rena. 1993. Germinação de sementes. In Aguiar IB, FCM Pinã-Rodrigues, MB Figliolia. Sementes florestais tropicais. Brasília, Brasil. ABRATES. p. 83-136.

Capelanes TMC, LC Biella. 1984. Programa de produção e tecnologia de sementes de espécies florestais nativas desenvolvido pela Companhia Energética de São Paulo - CESP. In Simpósio brasileiro sobre tecnologia de sementes florestais. Belo Horizonte, Brasil. p. 85-107.

Carvalho NM, J Nakagawa. 2000. Sementes. Ciência, tecnologia e produção. FUNEP. 588 p.

Castro MN. 2003. Influência de diferentes temperaturas na germinação de sementes de Tabebuia capitata (Bur. et $\mathrm{K}$. Schum. Sandw) Tesis en Biólogia. Manaus, Brasil. Instituto de Tecnologia de la Amazonía. Universidad de Manaus. 24 p.

Cavalcante AMB, SCSGA Perez. 1995. Efeitos da temperatura sobre a germinação de sementes de Leucaena leucocephala (LAM) de WIT. Revista Brasileira de Sementes 17(1): 1-8.

Copeland LO, MB Mc Donald. 1999. Principles of seed science and technology. London. England Kluwer Academic Publishers. 409 p.

Cruz CD. 2001. Programa genes. Versão windows. Aplicativo computacional em genética e estatística. Viçosa UFV, Brasil. 648 p. 
Ferreira AG, F Borghetti. 2004. Germinação de Sementes: Do básico ao aplicado.

Gomes SMS, RL Bruno. 1992. Influência da temperatura e substratos na germinação de sementes de urucum Bixa orellana L. Revista Brasileira de Sementes 14(1): 47-50.

Hand DJ, G Craig, M Takaki, R Kendrick. 1982. Interaction of light and temperature on seed germination of Rumex obtusifolius L. Planta 156(1): 457-460.

Hendricks SB, RB Taylorson. 1976. Variation in germination and amino acid leakage of seeds with temperature related to membrane phase change. Plant Physiology 58(1): 7-11.

Kraemer KH, NA Kampf, ME Áquila. 2000. Luz e temperatura na germinação de sementes de Tibouchina urvilleana. Revista Brasileira de Horticultura Ornamental 6(1/2): 39-45.

Labouriau LG. 1983. A germinação das sementes. Washington, USA. OEA. 174 p.

Labouriau LG, Pacheco A. 1978. On the frequency of isothermal germination in seeds of Dolichos biflorus L. Plant \& Cell Physiology 19(3): 507-512.

Lima CMR, F Borghetti, MV Sousa. 1997. Temperature and germination of the Leguminosae Enterolobium contortisiliquum. Revista Brasileira de Fisiologia Vegetal 9(2): 97-102

Lorenzi H. 1992. Árvores brasileiras: Manual de identificação e cultivo de plantas arbóreas nativas do Brasil. Nova Odessa, Brasil. Editora Plantarum. 368 p.

Maguire JD. 1962. Speed of germination: aid in selection and evaluation for seedling emergence and vigour. Crop Science 2(2): 176-177.

Mayer AM, A Poljakoff-Mayber. 1989. The germination of seeds. Oxford, UK. Pergamon Press. 270 p.

Ministério da Agricultura e Reforma Agrária. 1992. Regras para analise de sementes. Brasília, Brasil. 356 p.

Nakagawa J. 1999. Testes de vigor baseados no desenvolvimento das plântulas. In Krzyzanoswski FCH, RD Vieira, JB França Neto eds. Vigor de sementes. Conceitos e testes. Londrina, Brasil. ABRATES. p. 2-24.
Oliveira EC. 1993. Morfologia de plântulas florestais. In Aguiar IB, FCM Piña Rodrigues, MB Figliolia eds. Sementes florestais tropicais. San Pablo, Brasil. p. 175-214.

Paula RC. 2007. Repetibilidade e divergência genética entre matrizes de Pterogyne nitens Tul. Fabaceae-Caesalpinioideae por caracteres biométricos de frutos e de sementes e parâmetros da qualidade fisiológica de sementes. Tesis de Libre Docéncia en Silvicultura. Jaboticabal, Brasil. Faculdad de Ciencias Agrarias y Veterinárias, Universidad Estadual Paulista. 128 p.

Pereira TS, CS Andrade. 1994. Germinação de Psidium guajava L. e Passiflora edulis Sims- Efeito da temperatura, substrato e morfologia do desenvolvimento pós-seminal. Revista Brasileira de Sementes 16(1): 58-62.

Probert RJ, RD Smith, P Birh. 1986. Germination responses to light and alternating temperatures in European populations of Dactylis glomerata LV. The principle components of the alternating temperature requeriment. The New Phytologist 102(1): 133-142.

Salomão NA, MTS Eira, R Cunha. 1995. The effect of temperature on seed germination of four Dalbergia nigra Fr. Allem Leguminosae. Revista Árvore 9(4): 588-594.

Santos RGS, IB Aguiar. 2000. Germinação de sementes de Sebastiana commersoniana (Bill.) (Smith et Downs) em função do substrato e do regime de temperatura. Revista Brasileira de Sementes 22(1): 120-126.

Scott AJ, MA Knott. 1974. Cluster-analysis method for grouping means in analysis of variance. Biometrics 30:507-512.

Vazquez-Yanes C, A Orozco-Segovia. 1982. Seed germination of a tropical rain forest pioneer tree (Heliocarpus donnellsmithii) in response to diurnal fluctuations of temperature. Physiologia Plantarum 56(3): 295-298.

Vazquez-Yanes C, A Orozco-Segovia. 1984. Fisiología y ecología de semillas de árboles de la selva tropical. Un reflejo de su ambiente. Ciencia 35(1): 191-201.

Recibido: 01.05.09

Aceptado: 09.11.09 